\title{
Efeito do porta-enxerto sobre a concentração de compostos fenólicos em vinhos Cabernet Sauvignon e Merlot
}

\author{
The effect of rootstocks on the concentration of phenolic compounds in Cabernet \\ Sauvignon and Merlot wines
}
Ricardo Allebrandt ${ }^{1 \star}$, Marcelo Alves Moreira ${ }^{2}$, José Luiz Marcon Filho ${ }^{3}$, Douglas André Würz ${ }^{4}$, Betina Pereira De Bem ${ }^{5}$, Leo Rufato ${ }^{2}$, Adrielen Tamiris Canossa ${ }^{2}$

${ }^{1}$ Centro Universitário Dinâmica das Cataratas, Foz do Iguaçu, PR, Brasil. * Autor para correspondência: ricardoufsc@gmail.com

${ }^{2}$ Universidade do Estado de Santa Catarina, Lages, SC, Brasil.

${ }^{3}$ Vinícola Legado, Campo Largo, PR, Brasil.

${ }^{4}$ Instituto Federal de Santa Catarina, Canoinhas, SC, Brasil.

${ }^{5}$ Vinícola Villa De Bem, São Joaquim, SC, Brasil.

Submissão: 06/09/2018 / Aceite: 06/08/2020

\begin{abstract}
RESUMO
Há mais de um século, a viticultura deixou de ser a cultura de uma espécie ou uma variedade de videira, e passou a ser o cultivo de uma planta composta de dois genótipos: um para gerar o sistema radicular e o outro para produzir os frutos. Para se adaptar a essa nova realidade, a cadeia produtiva da uva e seus derivados tem desenvolvido estudos e pesquisas que vão desde o momento da enxertia até a degustação do vinho. Apesar do grande número de estudos sobre a influência dos porta-enxertos sobre o desempenho de variedades copa, muitos processos dessa interação ainda são desconhecidos. Este trabalho teve por objetivo avaliar o efeito de diferentes porta-enxertos sobre a concentração de compostos fenólicos de vinhos das variedades Cabernet Sauvignon e Merlot, produzidos no Planalto Sul de Santa Catarina, Brasil. Para tanto, foram testados, além do P1103, os porta-enxertos 3309C e 101-14Mgt. Os experimentos foram conduzidos nos municípios de Painel $\left(28^{\circ} 01^{\prime} \mathrm{S}, 50^{\circ} 08^{\prime} \mathrm{W}\right.$ e $\left.1.200 \mathrm{~m}\right)$ e São Joaquim (28¹4'S, 4958'O e 1.300 m), nas safras 2015, 2016 e 2017. Foram avaliadas as concentrações de compostos flavonoides e não-flavonoides nos vinhos. Os porta-enxertos influenciam na composição fenólica dos vinhos. O porta-enxerto 3309C aumenta a concentração de compostos flavonoides nos vinhos, em relação ao porta-enxerto tradicionalmente usado nos vinhedos do Planalto Sul de Santa Catarina.
\end{abstract}

PALAVRAS-CHAVE: Vitis vinifera, enxertia, flavonoides.

\begin{abstract}
Over a century ago, viticulture went from the cultivation of one species or grape variety to the cultivation of a plant composed of two genotypes: one responsible for growing the root system and the other for producing the fruit. To adapt to this new reality, a great amount of research has been done, with various studies ranging from grafting techniques to sensory analysis. Despite numerous studies on the influence of rootstocks on scion performance, many aspects of this interaction are still unclear. This study aimed to evaluate the effect that different rootstocks have on the concentration of phenolic compounds in Cabernet Sauvignon and Merlot wines produced in the Southern Highlands of Santa Catarina, Brazil, by testing rootstocks 3309C and 101-14Mgt, in addition to P1103. Experiments were conducted in the municipalities

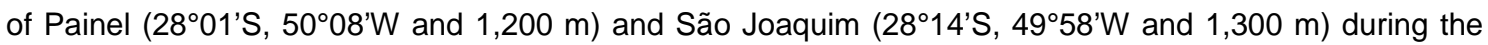
2015,2016 , and 2017 vintages to assess the concentrations of flavonoid and non-flavonoid compounds in the wines. The results indicate that different rootstocks affect the wines' phenolic composition. The concentration of flavonoid compounds increases with rootstock 3309C, compared to the commonly used rootstock P1103, in the Southern Highlands of Santa Catarina.
\end{abstract}

KEYWORDS: Vitis vinifera, grafting, flavonoids.

\section{INTRODUÇÃO}

Na maioria das regiões vitícolas do mundo, a produção de uvas europeias (Vitis vinifera) só é possível 
mediante a utilização de porta-enxertos obtidos a partir de espécies de videiras americanas (Vitis riparia, Vitis rupestres e $V$. berlandieri), ou a partir da hibridação entre elas. Essa realidade começou a ser conformada durante o final do século XIX, quando se descobriu que o uso de porta-enxertos era (e ainda é) a única forma eficaz de controlar a filoxera (Daktulospharia vitifoliae), um afídeo natural da América do Norte, que ataca as raízes de Vitis vinifera. Desde então, a viticultura deixou de ser o cultivo de apenas um genótipo, e passou a trabalhar com uma planta composta por dois genótipos: um para constituir o sistema radicular (porta-enxerto), e o outro para produzir os frutos (variedade copa). Para se adaptar a essa nova realidade, a cadeia produtiva da uva e seus derivados têm desenvolvido estudos e pesquisas que vão desde o momento da enxertia até a degustação do vinho. Apesar do grande número de estudos sobre a influência dos porta-enxertos sobre o desempenho de variedades copa, muitos processos dessa interação ainda são desconhecidos.

Um desses processos é a influência do porta-enxerto sobre a composição química dos vinhos. Sabese que as características de uma variedade de uva no momento da colheita são majoritariamente determinadas pela sua genética, e como ela interagiu com o ambiente durante o ciclo. Quando se pensa na expressão varietal nos vinhos, além dos fatores genéticos e ambientais, somam-se os fatores envolvidos no processo de vinificação (GONZALEZ-SAN JOSE et al. 1990). Nesse contexto, o porta-enxerto tem um papel importante, pois faz a conexão da variedade copa ao solo, o que o torna um elemento de adaptação ao ambiente (OLLAT et al. 2015), e pode indiretamente influenciar na expressão de características varietais da copa.

Os porta-enxertos vigorosos tendem a prolongar a maturação dos frutos da variedade copa, em comparação com videiras enxertadas em genótipos de baixo vigor, que por sua vez tendem a acelerar o processo de maturação (POUGET 1986). Porta-enxertos de baixo vigor podem diminuir o tamanho das bagas, aumentando a relação casca/polpa, o que resulta num aumento da concentração de compostos fenólicos e da cor dos vinhos (GIL et al. 2015). Além disso, se um porta-enxerto diminui o vigor da variedade copa, há uma diminuição no adensamento do dossel vegetativo, fazendo com que os cachos fiquem mais expostos à radiação solar, e estimulando o aumento da síntese de compostos fenólicos e de cor nas bagas (SPAYD et al. 2002, CORTELL \& KENNEDY 2006).

Nesse sentido, este trabalho teve por objetivo avaliar o efeito de diferentes porta-enxertos sobre a composição fenólica de vinhos das variedades Cabernet Sauvignon e Merlot.

\section{MATERIAL E MÉTODOS}

O experimento foi desenvolvido com uvas provindas de vinhedos comerciais localizados nos

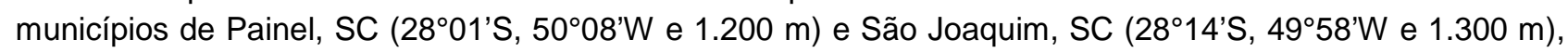
durante os ciclos 2014/2015, 2015/2016 e 2016/2017. Os vinhedos foram implantados em 2004 , nos espaçamentos de 3,0 m entre linhas e 1,5 m entre plantas. As plantas são conduzidas no sistema ípsilon ("Y") com poda em cordão esporonado duplo. Os solos da região enquadram-se nas classes Cambissolo Húmico, Neossolo Litólico e Nitossolo Háplico, desenvolvidos a partir de rocha riodacito e basalto. O clima da região, de acordo com o sistema de classificação climática de Köppen, é classificado como mesotérmico úmido e verão ameno (Cfb).

Os tratamentos consistiram da utilização de três porta-enxertos: Paulsen 1103 (P1103; V. berlandieri x V. rupestris), 3309 Couderc (3309C; V. riparia x V. rupestris) e 101-14 Millardet et de Grasset (101-14Mgt; $V$. riparia $x$ V. rupestris), avaliando-se seus efeitos na composição fenólica dos vinhos das variedades copa Cabernet Sauvignon e Merlot (Vitis vinifera). Para a variedade Cabernet Sauvignon, o experimento foi conduzido com uvas produzidas sobre os três porta-enxertos em Painel, SC, nas safras 2015, 2016 e 2017; e em São Joaquim, SC, nas safras 2015 e 2016. Para a variedade Merlot, o experimento foi conduzido com uvas produzidas apenas em Painel, SC, nas safras 2016 e 2017.

As variáveis analisadas foram os compostos fenólicos, flavonoides e não-flavonoides, dos vinhos de cada variedade, em combinação com os porta-enxertos e nas safras descritas acima. Os compostos flavonoides analisados foram: catequina, quercetina, rutina e caempferol. Os compostos não-flavonoides foram: ácido gálico, ácido vanílico, ácido p-coumárico e resveratrol.

Para a microvinificação, foram colhidas amostras de $40 \mathrm{~kg}$ de uva por tratamento, que foram mantidas em câmara fria por $24 \mathrm{~h}$ à temperatura de $5 \stackrel{\circ}{\circ}$. Os cachos passaram por desengaçadeira, separando-se as bagas das ráquis, e no recipiente em que recebeu as bagas se adicionou $60 \mathrm{mg} \mathrm{kg}^{-1}$ de $\mathrm{SO}_{2}$ a partir de uma solução com 10\% de metabissulfito de potássio. As bagas, de cada tratamento, foram divididas em quatro repetições de igual volume (aproximadamente $8 \mathrm{~kg}$ cada) e colocadas em fermentadores de plástico adaptados com batoque hidráulico. A inoculação de leveduras hidratadas ativas (Saccharomyces 
cerevisiae) foi realizada na proporção de $0,2 \mathrm{~g} \mathrm{~L}^{-1}$, quatro horas após as uvas terem sido colocadas nos fermentadores. A fermentação alcoólica ocorreu em uma sala climatizada com temperatura controlada a $20^{\circ} \mathrm{C}\left( \pm 2{ }^{\circ} \mathrm{C}\right)$. O tempo de maceração foi de sete dias, com duas remontagens diárias. Após a descuba, separação do vinho e do bagaço, o vinho foi trasfegado de duas a três vezes, até que se observou o fim da fermentação malolática. Uma das repetições foi utilizada para completar o volume dos fermentadores das outras três repetições, durante as trasfegas.

Finalizada a fermentação malolática, em cada repetição foram adicionadas $60 \mathrm{mg} \mathrm{L}^{-1}$ de $\mathrm{SO}_{2}$, mediante uma solução de metabissulfito a 10\%, e após isso, as repetições foram armazenadas em câmara fria, à temperatura de $0{ }^{\circ} \mathrm{C}\left( \pm 1^{\circ} \mathrm{C}\right)$ por 21 dias, para permitir a ocorrência da estabilização tartárica. Ao final, foram coletadas amostras do vinho pronto, em tubos Falcon de $50 \mathrm{ml}$, e armazenadas em congelador $\left(-18{ }^{\circ} \mathrm{C}\right)$ até $\mathrm{o}$ momento das análises em espectrofotômetro e em cromatografia líquida de alta eficiência (CLAE). O vinho restante foi envasado em garrafas de $375 \mathrm{ml}$ que foram armazenadas em sala climatizada a $18{ }^{\circ} \mathrm{C}$, para futuras análises de evolução em garrafa.

O delineamento experimental foi inteiramente casualizado, com quatro repetições. Sendo que cada repetição foi constituída de um fermentador. Para cada combinação de variedade copa e porta-enxerto, nos locais e safras já especificados, foram utilizadas quatro amostras $(50 \mathrm{ml}$ cada), provindas cada uma de um fermentador.

Para as análises dos vinhos, os compostos fenólicos foram quantificados em cromatografia líquida de alta eficiência de acordo com metodologia descrita por CADAHÍA et al. (2009) adaptada por FERREIRALIMA et al. (2013) com modificações.

Os reagentes utilizados na realização das análises como acetonitrila ( $\geq 99,9 \%$, Fluka), ácido acético $(\geq 99,7 \%$, Sigma -Aldrich) e metanol ( $\geq 99,8 \%$, Biotec) foram de grau cromatográfico. $O$ ácido $L(+)$ tartárico $(\geq 99 \%$, Vetec) e etanol ( $\geq 99,8 \%$, Vetec) foram de grau analítico. A água utilizada para as análises foi obtida através de destilação e posterior purificação Milli-Q, Sistema Simplicity UV (Millipore, Massachusetts, USA). Os padrões ácido gálico anidro $(\geq 98 \%),(+)$ - catequina ( $\geq 98 \%)$, ácido p-cumárico $(\geq 98 \%)$, ácido vanílico $(\geq 97 \%)$, resveratrol $(\geq 95 \%)$, quercetina $(\geq 95 \%)$, rutin $(\geq 94 \%)$ e campferol $(\geq 97 \%)$ foram obtidos na SigmaAldrich.

As soluções estoque de cada padrão foram preparadas em metanol e congeladas a $-18{ }^{\circ} \mathrm{C}$. Uma solução contendo uma mistura de todos os padrões foi preparada em sistema de vinho sintético $\left(5 \mathrm{~g} \mathrm{~L}^{-1}\right.$ ácido tartárico, $12 \% \mathrm{v} \mathrm{v}^{-1}$ de etanol e pH 3,2). O vinho sintético foi utilizado para evitar interferência na separação cromatográfica e na resposta de detecção. As soluções de calibração foram preparadas também em vinho sintético pela diluição da solução estoque contendo a mistura dos padrões. Todas as soluções utilizadas foram previamente filtradas em membrana com poros de 0,45 $\mu \mathrm{m}$ (Membrana PES-Kasvi). As faixas de calibração e equações para determinação dos compostos fenólicos estão descritas na Tabela 1.

Tabela 1. Curvas de calibração para a quantificação dos compostos fenólicos nos vinhos de Cabernet Sauvignon e Merlot por método de CLAE.

Table 1. Calibration curves for quantifying phenolic compounds in Cabernet Sauvignon and Merlot wines by the HPLC method.

\begin{tabular}{lllll}
\hline Composto & $\begin{array}{c}\text { Faixa de calibração } \\
\left(\mathrm{mg} \mathrm{L}^{-1}\right)\end{array}$ & $\begin{array}{c}\text { Equação linear } \\
\mathrm{y}=\mathrm{ax}\end{array}$ & $\mathrm{R}^{2}$ & $\begin{array}{c}\mathrm{TR} \\
\mathrm{min}\end{array}$ \\
\hline Ácido Gálico & $0.125-62.5$ & $\mathrm{y}=64889 \mathrm{x}$ & 0.9979 & 4.1 \\
Catequina & $0.125-158,8$ & $\mathrm{y}=17546 \mathrm{x}$ & 0.9992 & 25.4 \\
Ácido Vanílico & $0.125-62.5$ & $\mathrm{y}=40297 \mathrm{x}$ & 0.9999 & 28.9 \\
Ácido p-Coumárico & $0.125-62.5$ & $\mathrm{y}=114723 \mathrm{x}$ & 0.9999 & 38.2 \\
Rutin & $0.125-62.5$ & $\mathrm{y}=19623 \mathrm{x}$ & 0.9999 & 40.9 \\
Resveratrol & $0.125-62.5$ & $\mathrm{y}=86848 \mathrm{x}$ & 0.9971 & 53.6 \\
Quercetina & $0.125-62.5$ & $\mathrm{y}=31070 \mathrm{x}$ & 0.9848 & 59.7 \\
Caempferol & $0.125-62.5$ & $\mathrm{y}=40075 \mathrm{x}$ & 0.9829 & 68.6 \\
\hline
\end{tabular}

$\mathrm{R}^{2}=$ coeficiente de determinação; TR = Tempo de Retenção.

As amostras de aproximadamente $2 \mathrm{ml}$ (vinho ou solução de calibração) foram filtradas em membrana de 0,45 $\mu \mathrm{m}$ (Membrana PES-Kasvi) acoplada a uma seringa, e colocadas no vial para injeção direta no sistema CLAE. Foram utilizadas três amostras por tratamento (combinação variedade copa e 
porta-enxerto), provenientes do vinho congelado no momento do envase. Para cada amostra foi realizada a leitura em duplicata e quando se detectou variação > que 10\% realizou-se uma terceira leitura. A quantificação em $\mathrm{mg} \mathrm{L}^{-1}$ de todos os compostos foi determinada por curvas de calibração com padrão externo.

As análises cromatográficas foram realizadas utilizando um equipamento de cromatografia líquida de alta eficiência Shimadzu (Kyoto, Japão), equipado com um desgaseificador a vácuo (DGU-2A), sistema quaternário com bomba (LC-10ADVP), válvula (FCV-10ALVP), detector UV-VIS (SPD-10AV), auto injetor (SIL-10ADVP) e controlador (SCL-10AVP). A coluna foi a C18 (5 $\mu \mathrm{m}, 250 \mathrm{~mm} \times 4,6 \mathrm{~mm}$, Restek). O software utilizado para controlar o sistema gradiente, o detector e para aquisição dos dados foi Shimadzu Class-VP.

Utilizou-se gradiente com dois solventes A e B. Para a fase móvel A, utilizou-se água:ácido acético (98:2), e como solvente para fase móvel B água:ácido acético:acetonitrila (58:2:40). A eluição foi realizada através de gradiente linear, iniciando-se com $100 \%$ de solvente $A$; aos $55 \mathrm{~min}, 20 \%$ de solvente $A$ e $80 \%$ de solvente B; aos $70 \mathrm{~min}, 0 \%$ de $\mathrm{A}$ e $100 \%$ de B; e aos $80 \mathrm{~min}, 100 \%$ de A e $0 \%$ de B. O fluxo utilizado foi de $0,9 \mathrm{~mL} \mathrm{~min} \mathrm{~m}^{-1}$. Os compostos fenólicos foram lidos no comprimento de onda de $280 \mathrm{~nm}$. Todos os solventes utilizados como fase móvel foram previamente filtrados em membrana com poros de 0,45 $\mu$ m (Membrana PES-Kasvi).

Após as análises dos vinhos em CLAE e obtenção das concentrações dos compostos fenólicos em $\mathrm{mg} \mathrm{L}^{-1}$, os dados de cada variedade foram submetidos à análise de variância (ANOVA). Quando foram detectados efeitos significativos de porta-enxertos, procedeu-se o teste de comparação de médias de Fisher, ao nível de $5 \%$ de probabilidade de erro.

\section{RESULTADOS E DISCUSSÃO}

Os porta-enxertos influenciaram na concentração de todos os compostos fenólicos flavonoides de ambas variedades, com exceção da concentração de catequina nos vinhos de Cabernet Sauvignon de São Joaquim (Tabela 2) e nos vinhos de Merlot (Tabela 3). 3309C e 101-14Mgt aumentaram a concentração de catequina em relação a P1103, nos vinhos Cabernet Sauvignon de Painel. 3309 conferiu os maiores teores de quercetina e caempferol nos vinhos Cabernet Sauvignon de ambos locais. Os vinhos Cabernet Sauvignon de P1103 e 3309C tiveram os maiores teores de rutina em Painel, mas apresentaram os menores teores em São Joaquim.

Neste estudo, os compostos fenólicos analisados são categorizados em flavonoides e nãoflavonoides. Os flavonoides são os compostos responsáveis principalmente pela cor e adstringência dos vinhos, além de terem grande importância para a saúde humana (SANTOS-BUELGA \& SCALBERT 2000). Os flavonoides são subdivididos em três classes principais: os flavan-3-óis e seus derivados estruturais (proantocianidinas, ou taninos condensados), antocianinas e os flavonóis. Os porta-enxertos não exerceram influência consistente sobre a concentração de catequina, composto do grupo dos flavan-3-óis. Apenas no vinho Cabernet Sauvignon de Painel, em que foram observados maiores teores em 3309C e em 101-14Mgt, o que pode ser reflexo da diminuição do tamanho das bagas (dados não apresentados), causando um aumento da concentração de compostos flavan-3-óis nos vinhos. No vinho Cabernet Sauvignon de São Joaquim e no vinho Merlot não foram observadas variações atribuídas aos porta-enxertos.

A uva é um fruto não-climatérico e seu desenvolvimento ocorre em duas etapas de crescimento, separadas por uma fase lag (COOMBE 1976). Os compostos flavan-3-óis e seus derivados estruturais (proantocianidinas) são sintetizados durante a primeira fase do desenvolvimento das uvas, enquanto que as antocianinas são sintetizadas durante a segunda fase, que tem início a partir da virada de cor das bagas (BOSS et al. 1996, BOGS et al. 2006). A síntese de flavonoides também pode ocorrer em resposta a fatores externos como a radiação UV, seca e temperaturas baixas (CHALKER-SCOTT 1999, WINKEL-SHIRLEY 2002).

Em um estudo sobre o efeito do vigor sobre a composição de flavonoides em uvas e vinhos de Pinot Noir, os autores concluíram que o vigor da videira exerce maior influência no acúmulo de flavonoides nas cascas e menor ou nenhuma influência desses compostos nas sementes (CORTELL et al. 2005). A concentração de alguns flavan-3-óis e flavonóis nas cascas aumentou significativamente com a redução do vigor das videiras (CORTELL et al. 2005). Todavia, não ficou claro nesse trabalho se esse incremento foi consequência de algum mecanismo fisiológico causado pelo vigor em si, ou se o dossel menos vigoroso (dados não apresentados) proporcionou maior exposição dos cachos à luz e/ou calor. Dessa forma, os autores fizeram outro estudo, na mesma parcela de vinhedo, aplicando sombreamento nos cachos de plantas com baixo vigor e encontraram os mesmos resultados (CORTELL \& KENNEDY 2006). 
Tabela 2. Efeito de diferentes porta-enxertos na concentração de compostos flavonoides em vinhos Cabernet Sauvignon elaborados com uvas produzidas em Painel, nas safras 2015, 2016 e 2017; e em São Joaquim, nas safras 2015 e 2016.

Table 2. The effect of different rootstocks on the concentration of flavonoid compounds in Cabernet Sauvignon wines made with grapes grown in Painel in the 2015, 2016, and 2017 vintages and in São Joaquim in the 2015 and 2016 vintages.

\begin{tabular}{|c|c|c|c|c|c|c|c|c|c|c|c|c|c|}
\hline \multirow{2}{*}{ Variáveis } & \multirow{2}{*}{ Safra } & \multicolumn{12}{|c|}{ Porta-enxertos } \\
\hline & & \multicolumn{4}{|c|}{ P1103 } & \multicolumn{4}{|c|}{$3309 \mathrm{C}$} & \multicolumn{4}{|c|}{ 101-14Mgt } \\
\hline & & & & & ainel & & & & & & & & \\
\hline \multirow{4}{*}{$\begin{array}{l}\text { Catequina } \\
\left(\mathrm{mg} \mathrm{L}^{-1}\right)\end{array}$} & 2015 & 24,3 & \pm & 1,9 & $\mathrm{~b}$ & 32,3 & \pm & 5,6 & b & 49,0 & \pm & 7,0 & $\mathrm{a}$ \\
\hline & 2016 & 61,7 & \pm & 1,1 & a & 67,3 & \pm & 3,2 & a & 68,7 & \pm & 0,8 & $\mathrm{a}$ \\
\hline & 2017 & 45,6 & \pm & 0,7 & b & 67,6 & \pm & 1,4 & a & 65,6 & \pm & 2,2 & $\mathrm{a}$ \\
\hline & Média & 43,9 & \pm & 6,7 & b & 55,7 & \pm & 7,5 & a & 61,1 & \pm & 4,6 & a \\
\hline \multirow{4}{*}{$\begin{array}{l}\text { Quercetina } \\
\left(\mathrm{mg} \mathrm{L}^{-1}\right)\end{array}$} & 2015 & 4,5 & \pm & 0,3 & b & 4,1 & \pm & 0,3 & $b$ & 6,6 & \pm & 0,2 & $\mathrm{a}$ \\
\hline & 2016 & 4,4 & \pm & 0,0 & b & 4,5 & \pm & 0,2 & $b$ & 6,8 & \pm & 0,4 & $\mathrm{a}$ \\
\hline & 2017 & 24,5 & \pm & 0,1 & b & 28,2 & \pm & 0,0 & a & 17,5 & \pm & 0,0 & c \\
\hline & Média & 11,1 & \pm & 3,3 & b & 12,2 & \pm & 4,0 & a & 10,3 & \pm & 1,8 & c \\
\hline \multirow{4}{*}{$\begin{array}{l}\text { Rutina } \\
\left(\mathrm{mg} \mathrm{L}^{-1}\right)\end{array}$} & 2015 & $\mathrm{NQ}$ & \pm & - & - & $N Q$ & \pm & - & - & $N Q$ & \pm & - & - \\
\hline & 2016 & 2,0 & \pm & 0,1 & a & 1,9 & \pm & 0,1 & a & 2,1 & \pm & 0,2 & $\mathrm{a}$ \\
\hline & 2017 & 8,0 & \pm & 0,4 & a & 8,4 & \pm & 0,4 & a & 2,1 & \pm & 0,1 & b \\
\hline & Média & 5,0 & \pm & 1,4 & a & 5,1 & \pm & 1,5 & a & 2,1 & \pm & 0,1 & b \\
\hline \multirow{4}{*}{$\begin{array}{l}\text { Caempferol } \\
\left(\mathrm{mg} \mathrm{L}^{-1}\right)\end{array}$} & 2015 & 0,3 & \pm & 0,1 & c & 0,7 & \pm & 0,1 & b & 1,3 & \pm & 0,1 & $\mathrm{a}$ \\
\hline & 2016 & 0,4 & \pm & 0,1 & a & 0,1 & \pm & 0,0 & b & 0,2 & \pm & 0,1 & b \\
\hline & 2017 & 5,1 & \pm & 0,0 & b & 6,5 & \pm & 0,0 & a & 3,9 & \pm & 0,0 & c \\
\hline & Média & 1,9 & \pm & 0,8 & $\mathrm{~b}$ & 2,4 & \pm & 1,0 & a & 1,8 & \pm & 0,5 & c \\
\hline \multicolumn{14}{|c|}{ São Joaquim } \\
\hline \multirow{3}{*}{$\begin{array}{l}\text { Catequina } \\
\left(\mathrm{mg} \mathrm{L}^{-1}\right)\end{array}$} & 2015 & 63,1 & \pm & 13,3 & ns & 82,4 & \pm & 0,03 & - & 73,6 & \pm & 1,8 & - \\
\hline & 2016 & 71,6 & \pm & 7,2 & ns & 64,7 & \pm & 5,10 & - & 66,7 & \pm & 6,30 & - \\
\hline & Média & 67,4 & \pm & 2,50 & ns & 73,5 & \pm & 5,10 & - & 70,2 & \pm & 1,90 & - \\
\hline \multirow{3}{*}{$\begin{array}{l}\text { Quercetina } \\
\left(\mathrm{mg} \mathrm{L}^{-1}\right)\end{array}$} & 2015 & 11,3 & \pm & 0,6 & $b$ & 21,3 & \pm & 1,4 & a & 13,5 & \pm & 0,0 & b \\
\hline & 2016 & 0,8 & \pm & 0,0 & a & 0,4 & \pm & 0,0 & b & 0,3 & \pm & 0,0 & c \\
\hline & Média & 6,0 & \pm & 0,2 & b & 10,8 & \pm & 0,3 & a & 6,9 & \pm & 0,6 & b \\
\hline \multirow{3}{*}{$\begin{array}{l}\text { Rutina } \\
\left(\mathrm{mg} \mathrm{L}^{-1}\right)\end{array}$} & 2015 & $N Q$ & \pm & - & - & $N Q$ & \pm & - & - & $N Q$ & \pm & - & - \\
\hline & 2016 & 4,9 & \pm & 0,7 & b & 6,1 & \pm & 0,4 & b & 11,0 & \pm & 0,8 & a \\
\hline & Média & . & \pm & - & - & - & \pm & - & - & - & \pm & - & - \\
\hline \multirow{3}{*}{$\begin{array}{l}\text { Caempferol } \\
\left(\mathrm{mg} \mathrm{L}^{-1}\right)\end{array}$} & 2015 & 1,5 & \pm & 0,1 & b & 4,2 & \pm & 0,80 & a & 2,0 & \pm & 0,01 & b \\
\hline & 2016 & 0,10 & \pm & 0,02 & a & 0,07 & \pm & 0,01 & a & 0,03 & \pm & 0,01 & a \\
\hline & Média & 0,78 & \pm & 0,20 & $b$ & 2,13 & \pm & 0,40 & a & 1,02 & \pm & 0,10 & b \\
\hline
\end{tabular}

Médias com letras diferentes, na linha, diferem significativamente pelo teste LSD de Fisher $(p<0,05)$. Média \pm erro padrão. NQ = Não Quantificado. ns = não significativo.

Os teores de flavonóis foram representados pelos compostos quercetina, rutina e caempferol. Nos vinhos de Cabernet Sauvignon, os maiores teores de flavonóis foram observados quando essa variedade foi enxertada em 3309C, com exceção da rutina em Cabernet Sauvignon de São Joaquim. Já no vinho Merlot, os maiores teores de flavanóis foram encontrados em 101-14Mgt. Esses resultados podem estar relacionados com a diminuição do tamanho das bagas (GIL et al. 2015) e com a redução da área foliar e potencial diminuição do sombreamento dos cachos. Tem sido demonstrado que o sombreamento causa reduções significativas na concentração de flavonóis em uvas (SPAYD et al. 2002, DOWNEY et al. 2004) e maçãs (SOLOVCHENKO \& SCHMITZ-EIBERGER 2003). Os flavonóis constituem uma porção pequena dos compostos fenólicos do vinho, e sua quantidade é influenciada por fatores como a variedade, o processo de maceração e as condições climáticas (GONZALEZ-SAN JOSE et al. 1990, SPIORA \& GUTIERREZ GRANDA 1998). Apesar de sua baixa concentração, os flavonóis são importantes por participarem de processos de co-pigmentação com as antocianinas, alterando a cor dos vinhos e estabilizando os pigmentos (CHEYNIER \& RIGAUD 1986).

Nos vinhos de Cabernet Sauvignon, os compostos não-flavonóides foram pouco afetados pelos portaenxertos, sendo que a concentração de resveratrol foi maior em P1103 e as menores em 3309C e 10114Mgt, em Painel e em São Joaquim (Tabela 4). Em São Joaquim, 3309C não diferiu significativamente de P1103 quanto ao teor de resveratrol no vinho. A concentração de ácido gálico foi maior em 3309C e 10114Mgt apenas na área de São Joaquim. Nos vinhos Merlot, a maior concentração de ácido gálico foi observada em P1103, e a menor em 101-14Mgt (Tabela 5). 3309C aumentou os teores de ácido 
p-coumárico e de resveratrol. 101-14Mgt se equiparou a 3309C quanto aos teores de resveratrol, e ambos foram maiores que P1103.

Tabela 3. Efeito de diferentes porta-enxertos na concentração de compostos flavonoides em vinhos Merlot elaborados com uvas produzidas em Painel, nas safras 2016 e 2017.

Table 3. The effect of different rootstocks on the concentration of flavonoid compounds in Merlot wines made with grapes grown in Painel in the 2016 and 2017 vintages.

\begin{tabular}{|c|c|c|c|c|c|c|c|c|c|c|c|c|c|}
\hline \multirow{2}{*}{ Variáveis } & \multirow{2}{*}{ Safra } & \multicolumn{12}{|c|}{ Porta-enxertos } \\
\hline & & \multicolumn{4}{|c|}{ P1103 } & \multicolumn{4}{|c|}{$3309 \mathrm{C}$} & \multicolumn{4}{|c|}{$101-14 \mathrm{Mgt}$} \\
\hline \multirow{3}{*}{ Catequina $\left(\mathrm{mg} \mathrm{L}^{-1}\right)$} & 2016 & 15,1 & \pm & 0,7 & ns & 15,1 & \pm & 1,0 & - & 16,6 & \pm & 0,0 & - \\
\hline & 2017 & 51,6 & \pm & 0,1 & ns & 49,0 & \pm & 2,6 & - & 48,1 & \pm & 1,9 & - \\
\hline & Média & 33,3 & \pm & 8,2 & ns & 32,0 & \pm & 7,7 & - & 32,4 & \pm & 7,1 & - \\
\hline \multirow{3}{*}{ Quercetina $\left(\mathrm{mg} \mathrm{L}^{-1}\right)$} & 2016 & 1,5 & \pm & 0,1 & $\mathrm{a}$ & 1,3 & \pm & 0,1 & a & 1,4 & \pm & 0,1 & a \\
\hline & 2017 & 22,1 & \pm & 0,3 & b & 21,6 & \pm & 0,2 & b & 23,2 & \pm & 0,4 & a \\
\hline & Média & 11,8 & \pm & 4,6 & b & 11,5 & \pm & 4,5 & $b$ & 12,3 & \pm & 4,9 & a \\
\hline \multirow{3}{*}{ Rutina (mg L $\left.\mathrm{L}^{-1}\right)$} & 2016 & 1,3 & \pm & 0,7 & a & 1,0 & \pm & 0,1 & a & 1,5 & \pm & 0,1 & a \\
\hline & 2017 & 7,8 & \pm & 0,2 & b & 8,1 & \pm & 0,1 & b & 10,5 & \pm & 0,2 & a \\
\hline & Média & 4,6 & \pm & 1,5 & b & 4,6 & \pm & 1,6 & b & 6,0 & \pm & 2,0 & a \\
\hline \multirow{3}{*}{ Caempferol $\left(\mathrm{mg} \mathrm{L}^{-1}\right)$} & 2016 & 0,14 & \pm & 0,02 & a & 0,03 & \pm & 0,00 & a & 0,10 & \pm & 0,06 & a \\
\hline & 2017 & 1,66 & \pm & 0,04 & c & 1,80 & \pm & 0,01 & $\mathrm{~b}$ & 1,96 & \pm & 0,06 & $\mathrm{a}$ \\
\hline & Média & 0,90 & \pm & 0,34 & b & 0,91 & \pm & 0,39 & $\mathrm{~b}$ & 1,03 & \pm & 0,42 & a \\
\hline
\end{tabular}

Médias com letras diferentes na linha diferem significativamente pelo teste LSD de Fisher $(p<0,05)$. Média \pm erro padrão. ns = não significativo.

Tabela 4. Efeito de diferentes porta-enxertos na concentração de compostos não-flavonoides em vinhos Cabernet Sauvignon elaborados com uvas produzidas em Painel, nas safras 2015, 2016 e 2017; e em São Joaquim, nas safras 2015 e 2016.

Table 4. The effect of different rootstocks on the concentration of non-flavonoid compounds in Cabernet Sauvignon wines made with grapes grown in Painel in the 2015, 2016, and 2017 vintages and in São Joaquim in the 2015 and 2016 vintages.

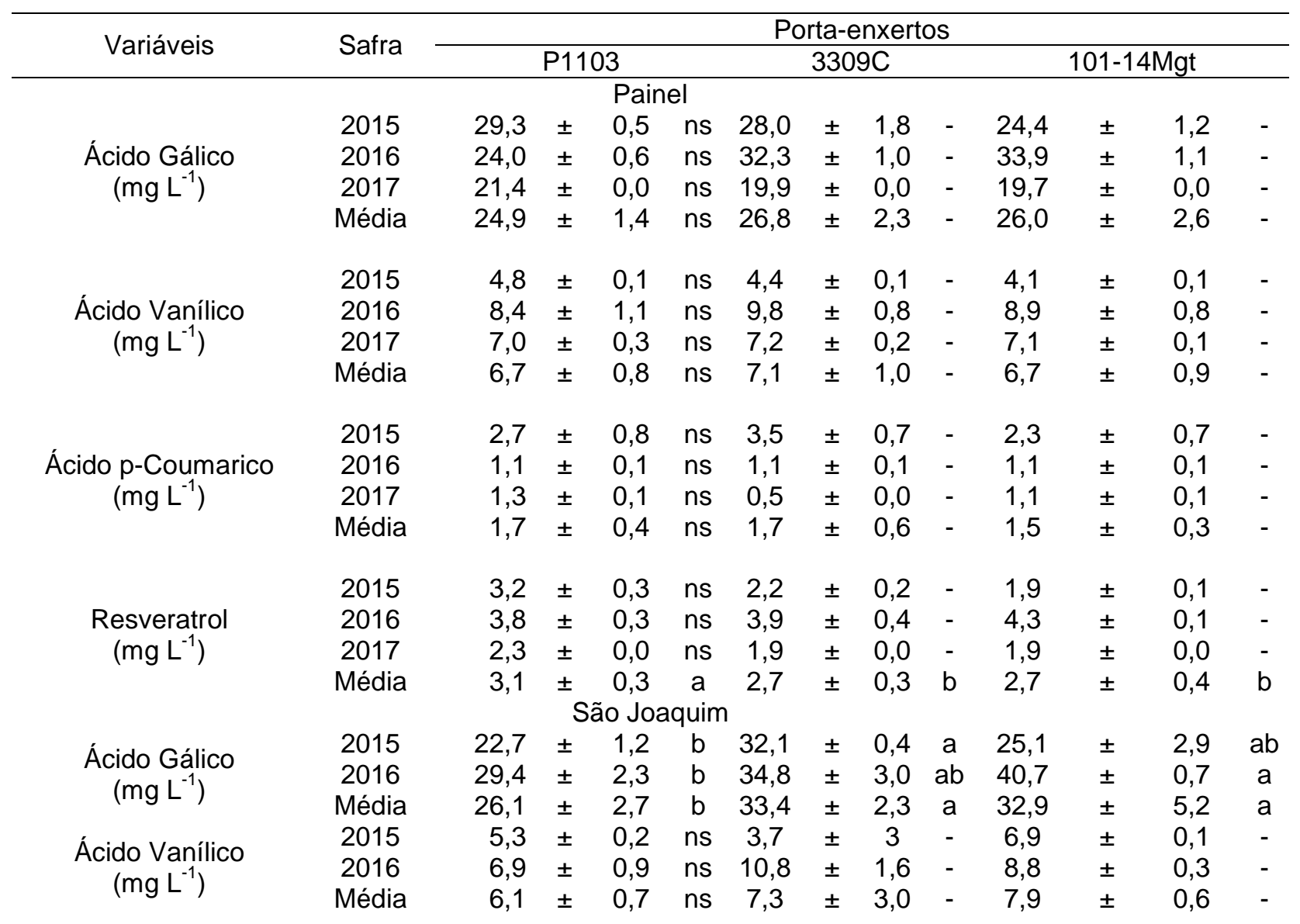




\section{Continuação Tabela 4....}

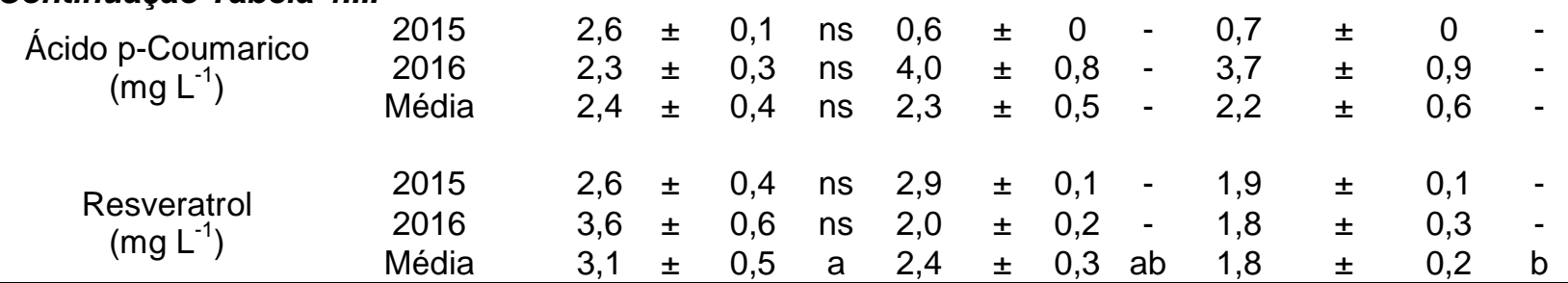

Médias com letras diferentes na linha diferem significativamente pelo teste LSD de Fisher $(p<0,05)$. Média \pm erro padrão. $n s=$ não significativo na ANOVA $(p<0,05)$.

Tabela 5. Efeito de diferentes porta-enxertos na concentração de compostos não-flavonoides em vinhos Merlot elaborados com uvas produzidas em Painel, nas safras 2016 e 2017.

Table 5. The effect of different rootstocks on the concentration of non-flavonoid compounds in Merlot wines made with grapes grown in Painel in the 2016 and 2017 vintages.

\begin{tabular}{|c|c|c|c|c|c|c|c|c|c|c|c|c|c|}
\hline \multirow{2}{*}{ Variáveis } & \multirow{2}{*}{ Safra } & \multicolumn{12}{|c|}{ Porta-enxertos } \\
\hline & & \multicolumn{4}{|c|}{ P1103 } & \multicolumn{4}{|c|}{$3309 \mathrm{C}$} & \multicolumn{4}{|c|}{$101-14$} \\
\hline \multirow{3}{*}{$\begin{array}{l}\text { Ácido Gálico } \\
\qquad\left(\mathrm{mg} \mathrm{L}^{-1}\right)\end{array}$} & 2016 & 21,9 & \pm & 0,1 & $\mathrm{a}$ & 20,2 & \pm & 0,0 & $b$ & 17,2 & \pm & 0,1 & C \\
\hline & 2017 & 22,5 & \pm & 0,1 & a & 21,7 & \pm & 0,0 & c & 22,1 & \pm & 0,1 & $b$ \\
\hline & Média & 22,3 & \pm & 0,2 & a & 21,0 & \pm & 0,3 & $b$ & 19,7 & \pm & 1,1 & C \\
\hline \multirow{3}{*}{$\begin{array}{l}\text { Ácido Vanílico } \\
\qquad\left(\mathrm{mg} \mathrm{L}^{-1}\right)\end{array}$} & 2016 & 4,6 & \pm & 0,4 & ns & 5,1 & \pm & 0,1 & - & 4,7 & \pm & 0,1 & - \\
\hline & 2017 & 5,8 & \pm & 2,9 & ns & 10,5 & \pm & 0,4 & - & 9,1 & \pm & 0,4 & - \\
\hline & Média & 5,2 & \pm & 1,3 & ns & 7,8 & \pm & 1,2 & - & 6,9 & \pm & 1,0 & - \\
\hline \multirow{3}{*}{$\begin{array}{c}\text { Ácido p-Coumarico } \\
\left(\mathrm{mg} \mathrm{L}^{-1}\right)\end{array}$} & 2016 & 4,6 & \pm & 0,0 & C & 5,3 & \pm & 0,0 & a & 4,9 & \pm & 0,1 & b \\
\hline & 2017 & 3,7 & \pm & 0,1 & $b$ & 4,8 & \pm & 0,1 & a & 3,7 & \pm & 0,0 & $b$ \\
\hline & Média & 4,1 & \pm & 0,2 & $C$ & 5,0 & \pm & 0,1 & a & 4,3 & \pm & 0,2 & $b$ \\
\hline \multirow{3}{*}{$\begin{array}{l}\text { Resveratrol } \\
\left(\mathrm{mg} \mathrm{L}^{-1}\right)\end{array}$} & 2016 & 3,4 & \pm & 0,1 & ns & 6,0 & \pm & 0,1 & - & 6,0 & \pm & 0,1 & - \\
\hline & 2017 & 10,6 & \pm & 0,8 & ns & 12,8 & \pm & 0,0 & - & 11,5 & \pm & 0,2 & - \\
\hline & Média & 7,0 & \pm & 1,7 & $b$ & 9,4 & \pm & 1,5 & a & 8,7 & \pm & 1,2 & a \\
\hline
\end{tabular}

Médias com letras diferentes na linha diferem significativamente pelo teste LSD de Fisher $(p<0,05)$. Média \pm erro padrão. ns = não significativo na ANOVA $(p<0,05)$

Os compostos não-flavonóides analisados neste trabalho são classificados como: ácidos hidroxibenzóicos (ácido gálico e vanílico), ácidos hidroxicinâmicos ( $p$-cumárico) e estilbenos (resveratrol). Para tais compostos, não foi observado um efeito consistente do porta-enxerto. Algumas diferenças foram detectadas, mas não se mantiveram ao longo das combinações de variedades copa, genótipos de portaenxerto e local. Dentre os ácidos hidroxibenzóicos, o teor de ácido gálico foi maior nos vinhos de Merlot no porta-enxerto P1103, em relação aos outros porta-enxertos. Mas nos vinhos de Cabernet Sauvignon de São Joaquim, foi observado o contrário, os porta-enxertos 3309C e 101-14Mgt induziram maior acúmulo de ácido gálico nos vinhos Cabernet Sauvignon. Sabe-se que os teores de ácidos hidroxibenzóicos e hidroxicinâmicos nas bagas aumentam à medida que a maturação avança e o teor de sólidos solúveis incrementam (TIAN et al. 2009). Neste sentido, os resultados encontrados neste trabalho estão de acordo, pois em São Joaquim, as uvas Cabernet Sauvignon atingiram teores de sólidos solúveis maiores em 101$14 \mathrm{Mgt}$, seguido de 3309C (dados não apresentados). O adiantamento da maturação, associado ao portaenxerto, em uvas viníferas já foi observado em estudo realizado com a variedade Merlot no Planalto Sul de Santa Catarina (ALLEBRANDT et al. 2015). Em um estudo realizado na Serra Gaúcha, no qual foram avaliados 15 porta-enxertos na maturação de Cabernet Sauvignon, os autores concluíram que o portaenxerto 101-14Mgt conferiu os maiores índices de maturação da uva (MIELE \& RIZZON 2019). JOGAIAH et al. (2015) encontraram os mesmos resultados em vinhos de Cabernet Sauvignon, quando as plantas eram enxertadas sobre 101-14Mgt.

Os ácidos hidroxicinâmicos e seus derivados participam como cofatores pigmentadores, portanto, uma maior proporção desses compostos nos vinhos elaborados com uvas de menor calibre poderia explicar parcialmente a intensa cor desses vinhos, por conta do efeito de copigmentação intermolecular (GIL et al. 2015). Em seu estudo sobre a influência do tamanho das bagas sobre a cor e a composição química do vinho, GIL et al. (2015) encontraram aumentos significativos nas concentrações de vários compostos fenólicos em vinhos feitos com bagas pequenas, de diâmetros menores que $11,5 \mathrm{~mm}$, em comparação com 
bagas médias e grandes $(11,5-14,5 \mathrm{~mm},>14,5 \mathrm{~mm}$, respectivamente).

\section{CONCLUSÃO}

Os porta-enxertos influenciaram na concentração dos compostos fenólicos flavonoides quercetina, rutina e caempferol nos vinhos das variedades Cabernet Sauvignon e Merlot.

O porta-enxerto 3309C aumentou a concentração da maioria dos compostos flavonoides nos vinhos de Cabernet Sauvignon, em relação ao porta-enxerto tradicionalmente usado (P1103), nos vinhedos do Planalto Sul de Santa Catarina.

Quanto aos compostos não-flavonoides, os porta-enxertos não exerceram efeito consistente nos vinhos de Cabernet Sauvignon e Merlot.

\section{AGRADECIMENTOS}

Às agências de financiamento CAPES, FAPESC e CNPq.

\section{REFERÊNCIAS}

ALLEBRANDT $R$ et al. 2015. Fenologia da variedade Merlot produzida sobre três porta-enxertos em elevadas altitudes de Santa Catarina. Revista Brasileira de Viticultura e Enologia 7: 36-46.

BOGS $\mathrm{J}$ et al. 2006. Identification of the flavonoid hydroxylases from grapevine and their regulation during fruit development. Plant Physiology 140: 279-291.

BOSS PK et al. 1996. Expression of anthocyanin biosynthesis pathway genes in red and white grapes. Plant molecular biology 32: 565-569.

CADAHÍA E et al. 2009. Chemical and chromatic characteristics of Tempranillo, Cabernet Sauvignon and Merlot wines from DO Navarra aged in Spanish and French oak barrels. Food Chemistry 115: 639-649.

CORTELL JM et al. 2005. Influence of vine vigor on grape (Vitis vinifera L. cv. Pinot noir) and wine proanthocyanidins. Journal of Agricultural and Food Chemistry 53: 5798-5808.

CORTELL JM \& KENNEDY JA. 2006. Effect of shading on accumulation of flavonoid compounds in (Vitis vinifera L.) pinot noir fruit and extraction in a model system. Journal of Agricultural and Food Chemistry 54: 8510-8520.

CHALKER-SCOTT L. 1999. Environmental significance of anthocyanins in plant stress responses. Photochemistry and Photobiology 70: 1-9.

CHEYNIER V \& RIGAUD J. 1986. HPLC separation and characterization of flavonols in the skins of Vitis vinifera var. Cinsault. American Journal of Enology and Viticulture 37: 248-252.

DOWNEY MO et al. 2004. The effect of bunch shading on berry development and flavonoid accumulation in Shiraz grapes. Australian Journal of Grape and Wine Research 10: 55-73.

FERREIRA-LIMA NE et al. 2013. Characterization of Goethe white wines: influence of different storage conditions on the wine evolution during bottle aging. European Food Research and Technology 237: 509-520.

GIL M et al. 2015. Influence of berry size on red wine colour and composition. Australian Journal of Grape and Wine Research 21: 200-212.

GONZALEZ-SAN JOSE $M$ et al. 1990. Anthocyanins as parameters for differentiating wines by grape variety, winegrowing region, and wine-making methods. Journal of Food Composition and Analysis 3: 54-66.

JOGAIAH S et al. 2015. Regulation of fruit and wine quality parameters of Cabernet Sauvignon grapevines (Vitis vinifera L.) by rootstocks in semiarid regions of India. Vitis 54: 65-72.

MIELE A \& RIZZON LA. 2019. Rootstock-scion interaction: 5. Effect on the evolution of Cabernet Sauvignon grape riepening. Revista Brasileira de Fruticultura 41: 1-10.

OLLAT N et al. 2015. Rootstocks as a component of adaptation to environment. In: GEROS H et al. (Ed.). Grapevine in a changing environment: a molecular and ecophysiological perspective. Nova Jersey: Wiley-Blackwell. p. 68-108.

POUGET R. 1986. Usefulness of rootstocks for controlling vine vigour and improving wine quality. In: Symposium on Grapevine Canopy and Vigor Management, XXII IHC 206. Leuven: ISHS Acta Horticulturae 206: 109-118.

SANTOS-BUELGA C \& SCALBERT A. 2000. Proanthocyanidins and tannin-like compounds-nature, occurrence, dietary intake and effects on nutrition and health. Journal of the Science of Food and Agriculture 80: 1094-1117.

SOLOVCHENKO A \& SCHMITZ-EIBERGER M. 2003. Significance of skin flavonoids for UV-B-protection in apple fruits. Journal of Experimental Botany 54: 1977-1984.

SPAYD SE et al. 2002. Separation of sunlight and temperature effects on the composition of Vitis vinifera cv. Merlot berries. American Journal of Enology and Viticulture 53: 171-182.

SPIORA MJ \& GUTIERREZ GRANDA MJ. 1998. Effect of pre-veraison irrigation cut off and skin contact time on composition color, and phenolic content of young Cabernet Sauvignon wines in Spain. American Journal of Enology and Viticulture 49: 153-161.

TIAN RR et al. 2009. Comparison of phenolic acids and flavan-3-ols during wine fermentation of grapes with different harvest times. Molecules 14: 827-838.

WINKEL-SHIRLEY B. 2002. Biosynthesis of flavonoids and effects of stress. Current Opinion in Plant Biology 5: 218223. 